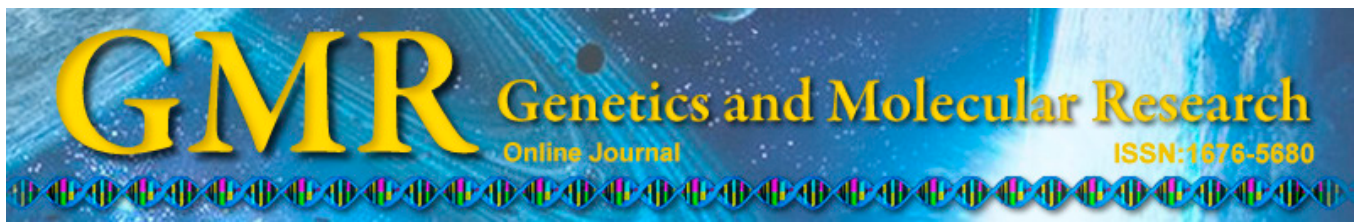

\title{
Short Communication \\ Isolation of microsatellite loci for Rhodiola alsia (Crassulaceae), an important ethno-medicinal herb endemic to the Qinghai-Tibetan plateau
}

\author{
F.Q. Zhang ${ }^{1,2 *}$, S.Y. Lei ${ }^{2 *}$, Q.B. Gao ${ }^{2}$, G. Khan ${ }^{2}$, R. Xing ${ }^{2}$, H.L. Yang ${ }^{2}$ and \\ S.L. Chen ${ }^{2}$ \\ ${ }^{1}$ Key Laboratory of Eco-Environments of Three Gorges Reservoir Region, \\ Ministry of Education, School of Life Sciences, Southwest University, \\ Chongqing, China \\ ${ }^{2}$ Key Laboratory of Adaptation and Evolution of Plateau Biota, \\ Northwest Institute of Plateau Biology, Chinese Academy of Sciences, \\ Xining, China \\ *These authors contributed equally to this study. \\ Corresponding authors: S.L. Chen / F.Q. Zhang \\ E-mail: slchen@nwipb.cas.cn / fqzhang@nwipb.cas.cn
}

Genet. Mol. Res. 14 (2): 5266-5269 (2015)

Received November 26, 2014

Accepted March 26, 2015

Published May 18, 2015

DOI http://dx.doi.org/10.4238/2015.May.18.18

\begin{abstract}
Rhodiola alsia, which has been used widely in traditional Chinese medicine for a considerable time, grows on moist habitats at high altitude near the snow line. Microsatellite loci were developed for $R$. alsia to investigate its population genetics. In total, 17 polymorphic microsatellites were developed based on ESTs from the Illumina HiSeq $^{\mathrm{TM}} 2000$ platform. The microsatellite loci were checked for variability using 80 individuals of $R$. alsia sampled from four locations on the Qinghai-Tibet Plateau. The total number of alleles per locus ranged from 10 to 20, and the observed heterozygosity ranged from 0.000 to 1.000 . The null allele frequency ranged from 0.000 to 0.324 . These microsatellites are expected to be helpful in future studies of population genetics in $R$. alsia and related species.
\end{abstract}

Key words: Rhodiola alsia; Simple sequence repeat; Population genetics; Qinghai-Tibet Plateau 


\section{INTRODUCTION}

The Qinghai-Tibet Plateau (QTP) is an important biodiversity hub for studies of evolution and speciation. Despite its importance, however, relatively little is known about its flora. To date, most molecular studies of QTP flora have used plastid DNAs and only a few have employed nuclear DNA markers (Liu et al., 2012). The insights that such studies provide into the genetic diversity and evolutionary history of species are of value in exploring the relationships between evolutionary and demographic histories (Avise et al., 1987). Rhodiola L. (Crassulaceae) consists of about 90 species, of which 20 are used in traditional Chinese medicine. Their therapeutic activities include preventing high altitude sickness and eliminating fatigue (Arora et al., 2005). Rhodiola alsia is a popular ethno-medicinal herb exclusively endemic to QTP. This species is found on moist habitats near the snow line at high altitude (Wu et al., 2001). In a previous study, 17 microsatellite markers were developed for 4 species of Rhodiola (You et al., 2013). However, the markers did not successfully amplify target sequences in $R$. alsia. In this study, we designed novel SSR (simple sequence repeat) markers for $R$. alsia based on microsatellite loci of biparentally inherited nuclear DNA. In total, 17 polymorphic SSR markers were isolated and characterized from expressed sequence tag (EST)-based sequences. These SSR markers are different from those developed previously with regard to sequence, repeat motifs and size (You et al., 2013). The microsatellite markers developed here will provide an efficient tool for population genetic studies and for assessing genetic diversity and spatial genetic structures at a larger scale in $R$. alsia and its close congeners.

\section{MATERIAL AND METHODS}

Eighty $R$. alsia individuals from four populations [Qingshuihe (18 individuals), Xiewu (21 individuals), Shiqu (18 individuals) and Dingqing (23 individuals)] were used in this study (Table 1). Fresh leaves were collected and dried using silica gel. Total genomic DNA was extracted using the modified cetyltrimethylammonium bromide method (Gao et al., 2012). Microsatellite markers were detected in $R$. alsia based on EST obtained using an Illumina HiSeq ${ }^{\mathrm{TM}} 2000$ platform (Illumina, San Diego, CA, USA). The SSRs were isolated by Microsatellite (MISA; http://pgrc.ipk-gatersleben.de/misa/) using Unigenes as reference. Only SSR lengths longer than $150 \mathrm{bp}$ upstream and downstream were used. SSR marker parameters were set to identify perfect mono-, di-, tri-, tetra-, penta-, and hexanucleotide motifs with minimum repeats of $12,6,5,5,4$, and 4, respectively. One hundred SSR loci were selected randomly from the identified loci. All the primers were designed by BGI-Shenzhen (Shenzhen, Guangdong, China) using the primer 3-2.3.4 software (http://primer3.sourceforge. net/). These primers were used to identify highly polymorphic loci in the 80 individuals from the four populations.

\begin{tabular}{|c|c|c|c|c|c|}
\hline Population code & Location & $\begin{array}{c}\text { Sample } \\
\text { size }\end{array}$ & Voucher No. & $\begin{array}{l}\text { Geographic } \\
\text { coordinates }\end{array}$ & $\begin{array}{l}\text { Altitude } \\
\text { (m) }\end{array}$ \\
\hline$\overline{\mathrm{QSH}}$ & Qingshuihe, Qinghai Province, China & 18 & Chen2002021 & $34^{\circ} 05^{\prime} \mathrm{N}, 97^{\circ} 37^{\prime} \mathrm{E}$ & 4670 \\
\hline XW & Xiewu, Qinghai Province, China & 21 & Chen 2002023 & $33^{\circ} 12^{\prime} \mathrm{N}, 97^{\circ} 26^{\prime} \mathrm{E}$ & 4350 \\
\hline SQ & Shiqu, Sichuan Province, China & 18 & Chen2002151 & $33^{\circ} 08^{\prime} \mathrm{N}, 97^{\circ} 33^{\prime} \mathrm{E}$ & 4390 \\
\hline DQ & Dingqing, Tibet Autonomous Region, China & 23 & Chen2002056 & $31^{\circ} 41^{\prime} \mathrm{N}, 94^{\circ} 55^{\prime} \mathrm{E}$ & 4900 \\
\hline
\end{tabular}

The voucher specimens are deposited in the Herbarium of the Northwest Institute of Plateau Biology (HNWP), Xining, Qinghai Province, China. 
Each polymerase chain reaction (PCR) was carried out using a $20-\mu \mathrm{L}$ reaction mixture. The PCR master mix contained $2 \mu \mathrm{L} 20-60$ ng template DNA, $2 \mu \mathrm{L}$ 10X PCR buffer, $0.6 \mu \mathrm{L} 2.5$ $\mathrm{mM}$ dNTPs, $0.5 \mu \mathrm{L}$ of each primer $(100 \mathrm{nM}), 0.2 \mu \mathrm{L}$ Taq polymerase (Takara, Dalian, China) and $14.2 \mu \mathrm{L} \mathrm{ddH_{2 }} \mathrm{O}$. The following amplification conditions were used: denaturation for $5 \mathrm{~min}$ at $94^{\circ} \mathrm{C}$; followed by 35 cycles of $45 \mathrm{~s}$ at $94^{\circ} \mathrm{C}, 30 \mathrm{~s}$ at the annealing temperature for each primer and $30 \mathrm{~s}$ at $72^{\circ} \mathrm{C}$; and, a final extension step of $7 \mathrm{~min}$ at $72^{\circ} \mathrm{C}$. The PCR products were amplified on agarose and polyacrylamide gels. Loci displaying high levels of polymorphism were then applied to a QIAxcel advanced system (QIAGEN, Hilden, Germany). The total number of alleles, observed heterozygosity, expected heterozygosity, null allele frequency, deviation from Hardy-Weinberg equilibrium, and linkage disequilibrium were calculated using the GENEPOP online package (http://genepop.curtin.edu.au/).

\section{RESULTS}

A total of 100 SSR loci were randomly selected for PCR amplification. Of these, 55 were successfully amplified, and 17 SSR loci proved to be highly polymorphic (Table 2). The total number of alleles per locus ranged from 10-20, with an average number of 14.8. The observed and expected heterozygosities ranged from 0.000-1.000 and 0.356-0.929, with an

\begin{tabular}{|c|c|c|c|c|c|c|}
\hline Locus & Primer sequences (5'-3') & Repeat motif & $\begin{array}{l}\text { Fragment } \\
\text { size (bp) }\end{array}$ & $\begin{array}{c}\mathrm{Ta} \\
\left({ }^{\circ} \mathrm{C}\right)\end{array}$ & $\begin{array}{l}\text { Total No. } \\
\text { of alleles }\end{array}$ & $\begin{array}{l}\text { GenBank } \\
\text { accession No. }\end{array}$ \\
\hline RA50 & $\begin{array}{l}\text { F: TTCCTCGGTAGCTATGATGATGT } \\
\text { R: TAAATGAGTATTGCTTTCGCCTT }\end{array}$ & $(\mathrm{AT})_{8}$ & 153 & 54 & 13 & KJ804158 \\
\hline RA49 & $\begin{array}{l}\text { F: TTGTGGAAATCTGTGTTGTTCAG } \\
\text { R: TTTCTTTCTGGGTTGTTTTTATCTG }\end{array}$ & $(\mathrm{AG})_{8}$ & 136 & 54 & 17 & KM657855 \\
\hline RA48 & $\begin{array}{l}\text { F: ACGCGTTGATGAGAGAGTGTTAT } \\
\text { R: ACCATCCAGCTAGCTAAGACCA }\end{array}$ & $(\mathrm{AG})_{7}$ & 138 & 56 & 10 & KJ804160 \\
\hline RA44 & $\begin{array}{l}\text { F: TTCTTCAATCTGGAACCTCCTC } \\
\text { R: GAGTCCTTTGACGACGAAAACTA }\end{array}$ & $(\mathrm{CT})_{7}$ & 136 & 56 & 20 & KM657854 \\
\hline RA41 & $\begin{array}{l}\text { F: GCATGATGACGGAGTAGGAGAT } \\
\text { R: CCTAACGTTCAATTTTCACCTCA }\end{array}$ & $(\mathrm{AG})_{7}$ & 142 & 56 & 15 & KJ804161 \\
\hline RA38 & $\begin{array}{l}\text { F: CTGTGGAGGATTTGGAAAATATG } \\
\text { R: CATACAAGCTTCATCCTTCCATT }\end{array}$ & $(\mathrm{AG})_{7}$ & 156 & 56 & 16 & KM657853 \\
\hline RA37 & $\begin{array}{l}\text { F: GAGATTCGGACTCGAAATTGG } \\
\text { R: CGCTCAACTTTATTCCTCCTCTT }\end{array}$ & $(\mathrm{GA})_{8}$ & 84 & 54 & 14 & KM657852 \\
\hline RA36 & $\begin{array}{l}\text { F: TCTGAACAGAAGATTTGTTTGGC } \\
\text { R: CGAAGAAAACAGAACTTTTGACG }\end{array}$ & $(\mathrm{GA})_{8}$ & 123 & 54 & 17 & KM657851 \\
\hline RA33 & $\begin{array}{l}\text { F: CCATACATATCGGACAGCTGAAT } \\
\text { R: TTGCAACTCGAAGAAAGACAAGT }\end{array}$ & $(\mathrm{AGG})_{7}$ & 110 & 56 & 13 & KM657850 \\
\hline RA32 & $\begin{array}{l}\text { F: CAATATTAACCTCACCCCCAATC } \\
\text { R: TCTCGTCATGGGTCCAGC }\end{array}$ & $(\mathrm{CT})_{9}$ & 159 & 54 & 19 & KM657849 \\
\hline RA23 & $\begin{array}{l}\text { F: TTCTTGTACAATTCAGGCCATCT } \\
\text { R: ATGATTATGTTCACCCTATGCCA }\end{array}$ & $(\mathrm{TG})_{8}$ & 143 & 56 & 13 & KJ804162 \\
\hline RA20 & $\begin{array}{l}\text { F: GCAGTGGATTGAAAAGATGAGAT } \\
\text { R: AAAAACGTGTCCAAGTCTCTCAC }\end{array}$ & $(\mathrm{TG})_{8}$ & 158 & 56 & 14 & KJ804163 \\
\hline RA19 & $\begin{array}{l}\text { F: TGTAGGAAAGTTGTTCCTTGTGG } \\
\text { R: CCTTTGATCTTTCAGCTCCTCTT }\end{array}$ & $(\mathrm{AC})_{8}$ & 132 & 56 & 14 & KJ804164 \\
\hline RA18 & $\begin{array}{l}\text { F: TACTGAATTTGGTGGAGGTTTTG } \\
\text { R: CTGGAGACTTCCCAGTACCTTCT }\end{array}$ & $(A G)_{8}$ & 152 & 56 & 14 & KM657848 \\
\hline RA17 & $\begin{array}{l}\text { F: GTCTCTCCTCACTGAAACGATGT } \\
\text { R: CAACCCCAATTTCAATTTTCTTA }\end{array}$ & $(\mathrm{CT})_{8}$ & 128 & 56 & 19 & KM657847 \\
\hline RA15 & $\begin{array}{l}\text { F: CTCCCTCTGCTCAAATTTTTGTT } \\
\text { R: TGCTAACCTCTCTACTCCAAGCA }\end{array}$ & $(\mathrm{AG})_{8}$ & 143 & 56 & 14 & KJ804165 \\
\hline RA14 & $\begin{array}{l}\text { F: GAGAACAAATGTCAAACCCAAAA } \\
\text { R: GGGTTAGATTTGTGTGTCGCTTA }\end{array}$ & $(\mathrm{CT})_{9}$ & 113 & 54 & 10 & KJ804159 \\
\hline
\end{tabular}

$\mathrm{Ta}=$ annealing temperature. 
average of 0.804 and 0.799 , respectively. The null allele frequency ranged from $0.000-0.324$, with an average value of 0.040 (Table 3).

Table 3. Results of initial primer screening in four populations of Rhodiola alsia.

\begin{tabular}{|c|c|c|c|c|c|c|c|c|c|c|c|c|c|c|c|c|}
\hline \multirow[t]{2}{*}{ Locus } & \multicolumn{4}{|c|}{ Population QSH $(\mathrm{N}=18)$} & \multicolumn{4}{|c|}{ Population XW $(\mathrm{N}=21)$} & \multicolumn{4}{|c|}{ Population SQ $(\mathrm{N}=18)$} & \multicolumn{4}{|c|}{ Population DQ $(\mathrm{N}=23)$} \\
\hline & $A$ & $H_{\mathrm{O}}$ & $H_{\mathrm{E}}$ & $N_{\mathrm{A}}$ & $A$ & $H_{\mathrm{o}}$ & $H_{\mathrm{E}}$ & $N_{\mathrm{A}}$ & $A$ & $H_{\mathrm{O}}$ & $H_{\mathrm{E}}$ & $N_{\mathrm{A}}$ & $A$ & $H_{\mathrm{o}}$ & $H_{\mathrm{E}}$ & $N_{\mathrm{A}}$ \\
\hline RA50 & 6 & 0.556 & 0.719 & 0.049 & 6 & 0.857 & $0.620^{*}$ & 0.000 & 4 & 0.889 & $0.554 *$ & 0.000 & 9 & 0.696 & $0.757^{*}$ & 0.049 \\
\hline RA49 & 2 & 0.000 & $0.356^{*}$ & 0.279 & 6 & 0.143 & $0.633^{*}$ & 0.300 & 11 & 0.667 & $0.759^{*}$ & 0.000 & 12 & 0.957 & $0.884 *$ & 0.000 \\
\hline RA48 & 9 & 0.611 & $0.756^{*}$ & 0.120 & 7 & 1.000 & $0.770^{*}$ & 0.000 & 7 & 0.944 & $0.767^{*}$ & 0.000 & 7 & 1.000 & $0.773^{*}$ & 0.000 \\
\hline RA44 & 10 & 0.889 & 0.889 & 0.000 & 13 & 0.952 & $0.913 *$ & 0.000 & 12 & 0.833 & 0.911 & 0.014 & 17 & 1.000 & 0.929 & 0.000 \\
\hline RA41 & 9 & 0.667 & $0.863^{*}$ & 0.094 & 10 & 0.952 & 0.80 & 0.048 & 10 & 0.833 & 0.88 & 0.010 & 14 & 0.870 & 0.923 & 0.042 \\
\hline A38 & 5 & 0.222 & $0.565^{*}$ & 0.221 & 10 & 0.810 & $0.862 *$ & 0.000 & 10 & 0.944 & 0.859 & 0.000 & 13 & 0.957 & 0.807 & 0.000 \\
\hline A37 & 5 & 0.944 & 0.679 & 0.000 & 10 & 0.952 & 0.854 & 0.000 & 8 & 1.000 & $0.827 *$ & 0.000 & 11 & 0.783 & 0.836 & 0.000 \\
\hline RA36 & 9 & 0.833 & $0.811 *$ & 0.000 & 8 & 0.857 & 0.787 & 0.012 & 8 & 0.882 & 0.779 & 0.000 & 15 & 0.957 & 0.873 & 0.000 \\
\hline RA33 & 6 & 0.471 & 0.633 & 0.098 & 8 & 0.500 & $0.788^{*}$ & 0.171 & 6 & 0.778 & 0.837 & 0.040 & 9 & 0.773 & $0.779^{*}$ & 0.031 \\
\hline RA32 & 11 & 0.722 & $0.756^{*}$ & 0.000 & 10 & 1.000 & 0.753 & 0.000 & 13 & 0.944 & 0.887 & 0.000 & 10 & 1.000 & 0.748 & 0.000 \\
\hline RA23 & 6 & 0.167 & $0.746^{*}$ & 0.324 & 11 & 0.905 & 0.858 & 0.017 & 9 & 0.611 & $0.878^{*}$ & 0.144 & 12 & 0.957 & 0.893 & 0.000 \\
\hline RA20 & 8 & 0.722 & 0.789 & 0.004 & 8 & 0.810 & 0.791 & 0.000 & 10 & 0.722 & $0.902 *$ & 0.080 & 10 & 0.870 & 0.802 & 0.009 \\
\hline RA19 & 8 & 0.667 & $0.790 *$ & 0.063 & 9 & 0.762 & 0.787 & 0.000 & 9 & 0.778 & 0.833 & 0.019 & 13 & 1.000 & 0.914 & 0.000 \\
\hline RA18 & 5 & 0.500 & $0.705^{*}$ & 0.131 & 8 & 1.000 & $0.772 *$ & 0.000 & 6 & 0.944 & $0.783 *$ & 0.000 & 13 & 0.870 & $0.917^{*}$ & 0.060 \\
\hline RA17 & 9 & 0.556 & $0.867 *$ & 0.151 & 14 & 1.000 & 0.893 & 0.000 & 11 & 1.000 & 0.888 & 0.000 & 14 & 1.000 & 0.876 & 0.000 \\
\hline RA15 & 9 & 0.944 & 0.844 & 0.000 & 13 & 1.000 & 0.921 & 0.000 & 11 & 1.000 & 0.898 & 0.000 & 9 & 1.000 & 0.853 & 0.000 \\
\hline RA14 & 8 & 0.944 & $0.805^{*}$ & 0.000 & 5 & 1.000 & $0.674 *$ & 0.000 & 7 & 0.889 & 0.817 & 0.053 & 7 & 0.391 & $0.679 *$ & 0.115 \\
\hline
\end{tabular}

$A=$ total number of alleles per locus; $H_{\mathrm{O}}=$ observed heterozygosity; $H_{\mathrm{E}}=$ expected heterozygosity; $N_{\mathrm{A}}=$ null allele frequency; $\mathrm{N}=$ sample size for each population. *Significant departure from HWE at $\mathrm{P}<0.01$. See Table 1 for population abbreviations.

\section{DISCUSSION}

This investigation successfully used EST from the Illumina HiSeq ${ }^{\mathrm{TM}} 2000$ platform to develop 17 novel and highly polymorphic SSR markers in $R$. alsia. These markers are expected to be of considerable value in our future studies on genetic variation and conservation in $R$. alsia and related species.

\section{ACKNOWLEDGMENTS}

Research supported by the National Natural Science Foundation of China (\#31270270, \#31200281 and \#31400322), West Light Foundation of the Chinese Academy of Sciences, and the International Scientific and Technological Cooperation Projects of Qinghai Province (\#2014-HZ-812).

\section{REFERENCES}

Arora R, Chawla R, Sagar R, Prasad J, et al. (2005). Evaluation of radioprotective activities of Rhodiola imbricata Edgew - A high altitude plant. Mol. Cell Biochem. 273: 209-223.

Avise JC, Arnold J, Ball RM, Bermingham E et al. (1987). Intraspecific phylogeography: the mitochondrial DNA bridge between population genetics and systematics. Annu. Rev. Ecol. 18: 489-522

Gao QB, Zhang DJ, Duan YZ, Zhang FQ, et al. (2012). Intraspecific divergences of Rhodiola alsia (Crassulaceae) based on plastid DNA and internal transcribed spacer fragments. Bot. J. Linn. Soc. 168: 204-215.

Liu JQ, Sun YS, Ge XJ, Gao LM, et al. (2012). Phylogeographic studies of plants in China: advances in the past and directions in the future. J. Syst. Evol. 50: 267-275.

Wu ZY, Peter HR and Hong DY (2001). Flora of China. Volume 8. Science Press Beijing, China \& Missouri Botanical Garden Press, St. Louis.

You JL, Liu WS, Zhao Y, Zhu YQ, et al. (2013). Microsatellite markers in Rhodiola (Crassulaceae), a medicinal herb genus widely used in traditional Chinese medicine. Appl. Plant Sci. 1: Doi: 1200219. 